\title{
Lexis as most local context: towards an SFL approach to lexicology
}

\author{
Lise Fontaine(10
}

Correspondence: FontaineL@cf.ac.uk Cardiff University, Cardiff, UK

\begin{abstract}
The world of lexis is vast and complex and it is generally accepted in psycholinguistics that it is represented as part of a large complex network. However, in systemic functional linguistics (SFL) modelling lexis has remained a relatively underdeveloped area of the theory. The ideas underpinning this paper stem from exploring the interface of context and lexicology, asking how SFL does and could handle lexis within the theory. Here the SFL concept of context is used to develop a similar account of lexis. The argumentation is based on the assumption that 'knowing about' context and 'knowing about' lexis is contained and maintained within a networked cognitive system. The common view of the relationship between context and lexis is generally one of disambiguation, frequently through collocation. However, I argue that there is more involved than that. In this paper, I use the SFL approach to context to establish the first steps towards an analogous approach to lexicology. The conclusion offered here is that it is theoretically plausible to draw on the dimension of instantiation, in a complementary way to delicacy, in order to model lexis as most local context, where the lexeme (or lemma) is modelled as meaning potential.
\end{abstract}

\section{Introduction}

It will be clear to readers who are well-versed in the literature of systemic functional linguistics (SFL) that the title makes reference to a well cited phrase within the theory, 'lexis as most delicate grammar', originating in Halliday's 1961 paper and developed by Hasan (1996). This paper presents a new perspective on lexis within SFL theory by pursuing its study from a different theoretical dimension, that of instantiation rather than delicacy. For Halliday (1961:272), delicacy is "the scale of differentiation, or depth in detail" and it is clear from Hasan (1996) and also Martin (1992) that lexis as most delicate grammar is pursued along this dimension, which runs through the lexicogrammatical stratum and is generally represented as a system network of options. As Martin (1992:278) explains, "looked at from the perspective of grammar lexis is not different in kind but simply different in generality." The least delicate options are extended in increasing differentiation within the network to the most delicate options.

As attractive as it may seem to model lexis as most delicate grammar, it is not without problems as will be discussed below. This paper introduces a new theoretical perspective on lexis in SFL theory by developing it within a different dimension, instantiation, which as will be shown, leads to a different approach - lexis as most local context. Looked at from the perspective of instantiation, lexis can be seen in terms of meaning potential and

(C) The Author(s). 2017 Open Access This article is distributed under the terms of the Creative Commons Attribution 4.0 International License (http://creativecommons.org/licenses/by/4.0/), which permits unrestricted use, distribution, and reproduction in any medium, provided you give appropriate credit to the original author(s) and the source, provide a link to the Creative Commons license, and indicate if changes were made. 
as I argue in this paper, through this lens, the SFL concept of 'context' can be extended to account for the relationship between a lexeme (or lemma) and its instantiation as a lexical item in text. This is a potentially exciting development since it can offer a bridge between the more textually oriented theory of SFL and the more lexically driven approaches to lexis as typically found within corpus linguistics.

The two dimensions mentioned above, delicacy and instantiation, provide complementary rather than contradictory approaches. Work on lexis within SFL is scarce as will be discussed below and as with most challenging areas, there is merit in tackling a problem from more than one angle. The realm of word study is the domain of lexicology and so it seems sensible to ask whether there is any common ground to be found between this more bottom up approach to the lexico-grammar and SFL's more top down approach. The point of departure and the nature of the concerns are very different. Lexicologists tend to start with the word as the unit of study and work outwards, where outwards can mean into the mind or lexicon (typically psycholinguistics and to some extent cognitive linguistics) or into (co-)text and beyond (typically corpus linguistics). Lexicology covers all areas of interest related to what we think of as the word from lexical semantics to etymology and morphology, including the general area of phraseology. When it comes to phraseology, connections to context offer a potential area of common interest to the two perspectives on the lexico-grammar. What they share to some extent is an interest in lexico-grammar and in data that focusses on "the occurrence of patterns which lie somewhere between abstract structures and individual lexical items or combinations of these" (Butler, 2013:206). The interface of context and lexicology spans the range of lexical studies from the more cognitive perspective to the more social perspective.

Given the focus within systemic functional linguistics (SFL) on language as sociallyoriented semiotic, it can sometimes seem that the study of context and the study of lexis are worlds apart but they are not. We tend to think, metaphorically, that the word is a small unit and language is a much bigger unit but perhaps this is not actually the case. As Mel'ćuk (1981:57) says, 'not only every language but every lexeme of a language is an entire world in itself'. The world of the word is vast and complex and it is generally accepted that it is represented as part of a large complex network. The 'knowing about' a context and the 'knowing about' a word is contained and maintained within an individual's networked cognitive system. In this sense, there is perhaps not as much difference between knowing about a context and knowing about a word as we might have thought. This is an especially provocative thought if we consider how the concepts of meaning potential and instantiation apply to both.

As we move forward in this discussion, I propose another metaphor for the study of lexis, that of the Tardis from the BBC science fiction television series Doctor Who. The Tardis is remarkable, not only because it can travel through time and space but precisely because it is bigger on the inside. We find, with the lexeme, multiple dimensions which challenge our linguistic notions of size and relativity. Halliday has developed not only a multi-functional approach to language but also a multi-dimensional one. Halliday and Matthiessen (2014:20) identify five dimensions: structure or syntagmatic order (rank); system or paradigmatic order (delicacy); stratification (realization); instantiation; and metafunction. Figure 1 illustrates how instantiation and delicacy can be represented as distinct dimensions.

In this paper, I will propose some ways in which lexis can be explored by examining the dimension of instantiation and considering how the SFL approach to context can 


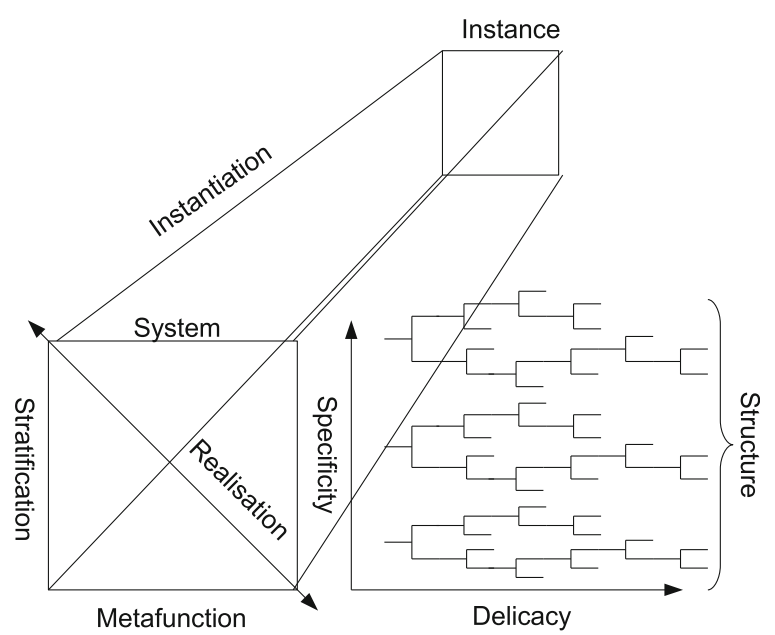

Fig. 1 Delicacy seen as a feature of system separate from instantiation and the dimensions of language (Wegener, 2011:88), reproduced here with permission

be applied to lexis. The main aim of the paper is to develop a new approach to the study of lexis within SFL. Drawing substantially from Halliday (1991) and Hanks (2013), I will use the SFL approach to context to argue for the need for an analogous approach to lexicology.

The paper will be organized as follows. The next section will situate lexicology in relation to SFL and outline the view of context adopted for the purposes of this paper, moving towards questions related to lexis and lexical representation in particular. Section 3 examines the current status of lexicology within the SFL framework and it argues for the need for a flexible lexicon (García Velasco, 2016). Section 4 considers the relationship between the meaning potential (Hanks, 2013) of a lexeme and the meaning of a word in use. Finally, section 5 closes the paper by addressing the question of whether it makes sense to talk about lexical meaning in SFL. I suggest that the framework for representing context could be applied by analogy to lexical representation. In this view, lexis is seen, not as most delicate grammar, but as most local context.

\section{Putting lexicology into context}

There is a deliberate play on words in the heading for this section. One part of the meaning relates to the frame, PUT X INTO CONTEXT, which means that X is given with the textual and/or situational context in which it was produced, i.e. provides more information so that $\mathrm{X}$ can be better understood. There is another related expression to this one, TAKE X OUT OF CONTEXT, which means that $\mathrm{X}$ has been removed from the textual and/or situational context in which it was produced, i.e. information is missing which makes understanding flawed in some way. In these two everyday expressions, we get a sense of what context suggests to most people and that is meaning or at least meaningful information. Context is where we 'get' meaning but it is also a kind of meaning, or rather kinds of meanings. In SFL terms, context "is that which helps determine meaning. This includes the surrounding text and the surrounding circumstances whatever they may be" (Wegener, 2011:4-5). The concept of meaning within SFL stems from Firth (1957), who saw meaning as function in context. For Halliday 
meaning is best viewed as 'choice' (Halliday, 2013), which he sees as an extension of Saussurian paradigmatic relations (Ibid.: 16).

The other part of the meaning of 'putting lexicology into context' concerns how lexicology can be viewed in relation to context, without restricting the notion of context to co-text or collocation. Normally SFL linguists interested in context and lexicologists generally are almost worlds apart. As suggested above, these are two perspectives that in many ways seem too distant to be merged. Certainly we do find research on context that relates to lexis and lexical analysis but this is not usually framed in lexicological terms which would be accessible to lexicologists. Similarly, it is true that lexicologists are increasingly interested in context (moving beyond co-text) but they do not generally adopt the SFL conceptualization of context.

In this section, I will first briefly outline the view of context taken in this paper and in the process draw on the key aspects for discussing the need to develop lexical representation within SFL.

Context is a specialized term in SFL, a theoretical metaphor, like the term 'choice', among others. Hasan (2013:298) argues that "[t]hese theoretical metaphors enable the analyst to enter into an explicit discourse on how language as a semiotic system becomes a powerful resource for the exchange of meanings in social contexts". By expanding context, as a theoretical metaphor, we can enable some important developments in terms of an SFL approach to lexicology or what we might simply call lexical representation.

Bartlett (2017: 375) explains context as "a unifying element within the overall architecture of SFL, linking language as system and instance (langue and parole) to the material conditions of those who use it". However, its definition and place within the theory is interpreted somewhat differently by different scholars (see for example Martin (1992), Butt et al. (2000), Matthiessen (2015), Wegener (2011), Bartlett (2017) and contributions by various authors in Fontaine et al., (2013), especially Bartlett (2013) and Bowcher (2013), to name only a few. At a more abstract level there is general agreement concerning some key points, including for example that context is accounted for by stratification and instantiation; that "[i]n exploring contextual and linguistic patterns in systemic functional linguistics, we try to interpret them in terms of dimensions" (Matthiessen, 2015:32) and that there are at least two strata of context, culture and situation. A full review of these positions is beyond the scope of this paper but see Bartlett (2017) for an overview of context. In this paper, the conceptualization of context is taken primarily from Halliday (1991), see Hasan (2009) which examines the place of context in the SFL framework, including an excellent discussion of Halliday's 1991 view of context and language.

The two aspects of context are explained by Halliday (1991: 271) as follows:

Language considered as a system - its lexical items and grammatical categories - is to be related to its context of culture; while instances of language in use - specific texts and their component parts - are to be related to their context of situation. Both these contexts are of course outside of language itself.

Context itself is not 'out there' as in outside the speaker. While it might be considered to be outside the language system, it cannot be denied that whatever context is, it 
is connected to or can interact with the language system. Halliday (1991:281) explains the connection as follows:

How do you set about "creating" a context for language? You cannot do it by means of legislation, like decreeing that poems are to be written in praise of a national leader. The only way is for the text itself to create its own context of situation.

This suggests that textual, i.e. linguistic, features are meaningful and that context of situation is effectively the collection of instances of text. Halliday (1991:277) sees this context as "a theoretical construct for explaining how a text relates to the social processes within which it is located". It is well established in SFL literature that this construct involves three variables or components, commonly termed Field, Tenor and Mode of Discourse, respectively as "the underlying social activity, the persons or "voices" involved in that activity, and the particular functions accorded to the text within it" (ibid.).

The difference between context of culture and context of situation according to Halliday (1991) is one of perspective; this difference is not meant to suggest different objects of study. Halliday (1991:276) explains this as follows:

These also are not two different things; they are the same thing seen from different points of view. A situation, as we are envisaging it, is simply an instance of culture; or, to put it the other way round, a culture is the potential behind all the different types of situation that occur.

Context of culture differs in its relationship to language since it is not tied to a context of situation through text but rather captures its potential. According to Halliday and Matthiessen (2014:32), "a given language is thus interpreted by reference to its semiotic habitat".

The relationship of context and language through realization and instantiation is shown in Fig. 2, taken from Halliday (1991).

Matthiessen (2015:34) explains that in Fig. 2, "situation type has the same location as context of culture (and context of situation) in terms of the hierarchy of stratification, but they are instead differentiated in terms of the cline of instantiation: context of culture is located at the potential pole, context of situation at the instance pole".

According to Hasan (2009:169), "instantiation is the relationship between a potential and its instance, context of culture is the potential, i.e. the system, while context of situation is an instance of that potential". The role of frequency (often described as probability in SFL) is important to the understanding of system and instantiation since it has a mediating role between system and instance. In theory, every instance perturbs the system to some degree or another, however (in)significant this may be. Plum (1998:31) states that the premise that "the realisation of context in text is probabilistic provides not only the key to making the hypothesis of the functional determination of text by its context testable but also provides a way of accounting for the variability found in text". This is a now common view within SFL but it leads to viewing lexis or at least lexicogrammatical structure as deterministic. It is at some level difficult to reconcile this view with Halliday's (1991:282) claim that "text and situation come into being together; so whatever kind of order we set up between them, it must be such that 


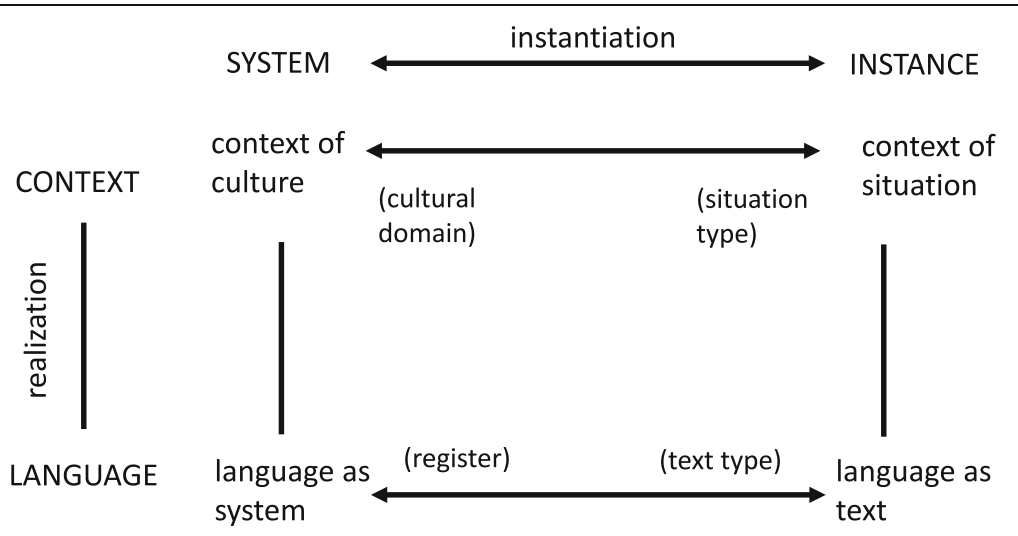

Fig. 2 Language and context, system and instance (adapted from Halliday, 1991:275)

we can start from either end". One way to view this relationship is to consider, as suggested by Tucker (2006:960), that "the full potential of language is reduced under the influence of the context of culture and the context of situation", which maintains the relationship between meaning potential and instance.

The relationship between context and lexicogrammatical systems is still being developed within SFL. For the position taken in this paper, the relationship of instantiation may provide one way to develop an account of lexical representation within the framework. The concept of construal is also significant. Halliday (1991:282) explains that "the culture is construed by systems of language choice; the situation is construed by patterns of language use". Based on Fig. 2, this statement can be rephrased in more abstract general terms as follows: the meaning potential is construed by systems of language choice; the instance is construed by patterns of language use. This is a point we will come back to in the next section when considering meaning potential and instance in terms of lexical representation.

\section{On lexicology and the SFL framework}

Lexicology concerns the study of words and their semantics, morphology, development and lexical representation, including word formation processes and the various types of relations among words. Context will be significant to lexical studies in different ways depending on the area of interest. For example, research into word histories requires a historical context, a diachronic perspective, often referred to as phylogenesis, and work related to the disambiguation of word senses involves context of situation and collocation. Areas that do not tend to overlap with SFL include lexical representation (i.e. what is its semantic nature and how is it represented) which covers morphology, semantics and lexical relations.

There are several research topics in SFL that do come close to a lexicological perspective. The first will be obvious to those familiar with the work. Cohesion in English (Halliday and Hasan, 1976) is the greatest contribution so far in terms of developing an approach to lexicology in SFL. This seminal work was ahead of its time when first published and remains an important source in any work related to cohesion. While it moved the field forward and was in many respects "ahead of its time" (Sanders and Maat, 2006:592), it does not address issues of concern to lexicologists. For example, on what 
basis is there a lexical tie between two items, i.e. what is the nature of the relationship? In some cases, lexical cohesion relies on an assumption that "a shared lexico-semantic relationship holds" (ibid.:591). This suggests that, for lexicologists, a more detailed account of lexical representation is needed for the SFL account of lexical relations to be more robust and usable in other approaches.

Martin's (1992) work in this area is noteworthy, in particular chapter five in English Text draws out some important issues and offers a very useful overview of how lexical relations can be approached within SFL. In particular, he discusses very well the "problem of units" (1992:290), which raises important questions about lexical representation. Indeed, as I do here, he asks how we can define lexical items. Interestingly, he proposes an alternative, suggesting it would be fruitful to "turn to the level of context" (p.291) and he shows that it is, but his interests and the types of questions he seeks to address lead the discussion towards register variables. This is not in any way a criticism since this perspective brings with it important insights. However it nevertheless remains concentrated on system networks and therefore the dimension of delicacy. Most approaches to lexis within SFL adhere to the dimension of delicacy. Martin (2016: 46-47) points out important contributions to studies of lexis in the area of phraseology, e.g. Tucker (2006) and Bednarek (e.g. 2008), where the focus is on corpusbased approaches to lexis; both make significant contributions to phraseology.

It is also worth mentioning work done on lexis within an SFL framework on verb sense classification. For example, Matthiessen (2014) and Thompson (2015) have both offered advances on lexical issues within transitivity. Discussions of transitivity configurations in SFL come very close to describing lexical representation for a given verb is typically the case in other approaches to transitivity. Finally, while there is no space here to discuss lexical modelling in computational approaches within SFL, it is worth noting very briefly work done by Fawcett, Tucker, and Lin, 1993; Fawcett, 1994; Matthiessen and Bateman, 1991; and O'Donnell, Cheng and Hitzeman, 1998 to name only a few. One important insight coming out of this work suggests there is a need for lexical representation of one kind or another, at least for computational applications.

While the approaches to lexis mentioned above certainly offer important insights to our understanding of lexis and lexico-grammar, they generally do not discuss in detail issues related to lexical representation or provide an account of what might be needed in terms of a lexical entry in the lexicon. As Martin (2016:45) points out:

Firth's interest in collocation ('the company words keep') has been developed in corpus linguistic research - chiefly by Sinclair and his colleagues (e.g. 1966; 1991) but less so in SFL where Halliday's early acknowledgement of the distinction between words and lexical items and the need for a syntagmatic perspective on expectancy relations among lexical items has tended to be backgrounded in relation to an interest in lexis as delicate grammar (the 'grammarian's dream', pursued by Hasan, and others).

It is often assumed that as the system networks become more delicate in terms of systemic choice, we find lexis in the most delicate systems. However, this is problematic in a number of ways. It is difficult to see how a lexeme could be represented as a choice 
in the system networks (although see Fawcett, 1994). The term 'lexeme' is used here in contrast to 'lexical item' in order to distinguish between an abstract entry in the mental lexicon (lexeme) and an instance of a lexeme in use (lexical item). However, the question is whether SFL needs to model lexemes or not. Questions of lexical representation have not been explicitly explored in detail, although Tucker's work (1996, 1998, 2006, 2009) comes very close. The position of lexis in Systemic Functional Linguistics (SFL) is, theoretically, fully integrated into the grammar if we accept the theory's assumption that "there is no need to postulate a separate 'lexicon' as a pre-existing entity on which the grammar is made to operate" (Halliday and Matthiessen, 1999: 199). If this is the case, it leaves open the question about where the lexical items are. One interpretation of lexis as most delicate grammar is that they are somehow the end point of a bundle of system choices, which leads to a single lexical option.

Hunston and Francis (2000:28) argue against this view:

If words have their typical phraseologies, such that words are not selected in isolation but in variable phrases, then it is unsatisfactory to propose that each lexical item is the end-point of an individual bundle of systemic choices. The best compromise that could be reached would be to propose that each bundle of system choices should end, not in a lexical item per se but in a 'unit of meaning'. As units of meaning are, by their nature, indeterminate in extent, however, such an interpretation would involve system-choices leading to fuzzy-edged and overlapping units, at best.

However, these positions are not necessarily entirely opposing. Butler (2009:59) suggests that "since a lexical item is defined by the selection expression through which it is generated, it could be claimed that there is in effect a kind of lexicon, and furthermore that the realisation statements attached to networks will specify the syntactic patterns associated with lexical items". In this sense, there may need to be some kind of lexicon, a large, complex network of lexical entries (however they are defined). The lexical items must be somewhere and must be accounted for somehow in the model. It is perhaps worth noting that Fawcett $(1994,2014)$ describes modelling nouns in the computational implementation of his model of SFL. The representation is in the form of an ontological network but there is no examination of the nature of the lexical item itself, i.e. in terms of its form(s) and functions or its semantic properties. Due to limitations of space, a full discussion of this is not possible here. On the issue of lexis discussed at this point, Fawcett concludes that "Halliday's original 1961 insight was well-founded, the only major modification needed being that lexically realized meanings are not necessarily most delicate', in the sense of 'at or near the terminal leaves of the system network" (1994:79).

Cognitive studies, including evidence from psycholinguistics, seem unanimous in accepting the need for a mental lexicon and one that plays an important role in both language production and language understanding according to Butler (2009:59). He goes on to say that "in language production the activation of an item from the lexicon must be accompanied, or at least closely followed, by the pairing of that item with a syntactic configuration which, in combination with the lexical item, begins to realise the conceptual structure being expressed. This is clearly easier in a model which contains a lexicon than in one which does not" (ibid.). The question remains then 
whether something could be gained (or lost) by making lexical representation in terms of a mental lexicon more explicit in SFL.

As an example of the need for more explicitness on lexical representation, let's consider the treatment of phrasal verbs, a type of multi-word expression (MWE), see Moon (1997) for a discussion of the many types of MWEs. Halliday and Matthiessen (2014:413) define them as "lexical verbs which consist of more than just the verb word itself". Fawcett (2000) has a similar position but differs in how these forms are represented in the theory. He considers that the lexical items are effectively separate elements of the clause (i.e. main verb and main verb extension) but that these forms jointly realize the process. If phrasal verbs are single lexical items, although orthographically expressed as two, then they should be analysed in the same way as any other multi-morphemic lexical item, such as unhappiness or understand. The issue of their potential discontinuity is not necessarily a barrier to this approach. Evidence from neurolinguistics by Cappelle, Shtyrov \& Pulvermuller (2010:200) supports the position "that language users store prefabricated chunks of lexical material which consist of more than one word and which can potentially be separated (e.g. heat the room up)". If this is how these MWEs are stored, then grammatical description should reflect this and the theoretical framework should represent them as such. In other words, lexico-grammatical description should be, at least in part, based on (or include) what we know about the cognitive aspects involved, e.g. how linguistic items are stored.

Within SFL, very little work has been done on what constitutes a word and it is often the case, as it is generally in linguistics, that the orthographic word is the default but this is problematic for a variety of reasons. The most important reason is because English orthography has not been consistent (into but out of, corkscrew but tea towel, etc.). Indeed, the very nature of what is a word cannot be taken for granted (see Wray 2014). The focus on the orthographic word is a real danger to studies of lexis and as corpus linguistics increases in popularity, it becomes even more important to challenge the assumptions surrounding the identification of lexical items. The status of the lexeme and indeed lexical representation within the theory is critically important, not just for the theory itself but for the areas it ventures into including applications and dialogue with other theories of language.

The call to develop work in this area can be traced back to Halliday's 1961 paper, where he put forward the ground-breaking idea that "the 'lexical item,' is unrestricted grammatically; grammatical categories do not apply to it, and the abstraction of the item itself from a number of occurrences ... depends on the formal, lexical relations into which it enters" (1961:277). At that time, he expected that "it should not be long before we find out much more about how language works at this level" (ibid.), since working out large scale frequencies of items in collocation would no longer be difficult. However, we have not yet seen this kind of detailed work, perhaps with the exception of Tucker (e.g. 1996).

As mentioned above, Martin points out the backgrounding of lexis in SFL, suggesting that this is related to an account of lexis as most delicate grammar. This view of lexis brings with it some degree of risk since it could result in treating lexis as taxonomically organised and this could lead to prioritising homophonous lexical relations rather than semantic ones. The reason for this is that if we take as a starting point Halliday's (1972/2013:8) view that "the output of any path through the network of systems is a structure", the 'most' delicate system will lead directly to a lexical item (cf criticisms by Hunston and Francis, 
2000 and Groom, 2005). This is a view of lexical representation which would be very difficult to accept for most lexicologists as it would predict that a lexeme with polysemes would need to be available at the terminal point of a variety of systems.

However, the nature of lexical representation is not clearly articulated in SFL. As Davidse (2017:79) makes very clear, "linguistic theories specify one's fundamental assumptions about language and the nature of the linguistic sign. It is within these assumptions that the facts of a language are described - that is, its categories identified and interpreted". The preference in lexical representation for homonymy or polysemy is not theory neutral, there are assumptions related to each position. SFL needs to offer detail on multiword expressions (MWEs) and on polysemy vs homonymy. There are some issues that need to be resolved if we want to claim as Hasan does that realization mediates between networks and structures (Hasan, 1996:74) since as concerns lexis, this suggests that a single lexical item is "the expression of a set of choices made in the system network" (Halliday, 1972/2013:8). The close connection of delicacy and paradigmatic arrangement can only take us so far; e.g. if lexical meaning is in part at least in its collocational associations or frames, it is difficult to think of lexical meaning as having paradigmatic relations. Even the definition of word and lexical item or the distinction between lexical/content words and grammatical/function words are areas which are not yet fully explained in terms of lexical representation.

In other functional approaches, the need for flexibility in lexical representation is being identified. For example, more flexible lexical representations and flexible lexicon (García Velasco, 2016), flexible word classes (Rijkhoff and van Lier, 2013) and fluid grammar (Steels and De Beule, 2006 and Steels, 2011). Indeed, cognitive models also suggest the need for a more flexible grammar. Pickering and Garrod (2004:21) state that their model of interactive alignment "challenges linguists to come up with a more flexible account of grammar capable of capturing linguistic constraints on linked sentence fragments". While a detailed look at flexible models is beyond the scope of this paper, these approaches have influenced my thinking on this topic. In the next section I will argue for a loosening of semantics in terms of lexical representation to allow for the meaning potential (Hanks, 2013) of a lexeme instantiated by a lexical item.

\section{Context, co-text and collocation: Where do (new) meanings come from?}

Since within the SFL framework, grammar and lexis are considered, as mentioned above (cf. Martin, 1992), as in essence the same but differentiated by degrees of generality, it is difficult to talk about lexical meaning outside of grammar. However, while I would agree this is not effectively possible since every lexical item is instantiated in text and therefore is fully integrated in the grammatical construction in which it appears. However, if we consider the possibility that the lexical item construes the meaning potential of the lexeme, then we can begin to see a way to capture lexical meaning through the lens of context, even if this is by analogy.

The main reason for being be concerned with lexical representation relates to the role of lexical classification in descriptions of lexicogrammatical structure. It is generally agreed that structural units tend to take shape around 'head' items. For example, in analysis, we distinguish the nominal group from the verbal group in terms 
of the class of item functioning as the head element of the unit. The label of the lexical item (e.g. noun or verb) is not so important (cf. Fawcett, 2000). Nevertheless, the classification of lexis and of units on the rank scale runs through grammatical description and indeed many key concepts in SFL theory, such as grammatical metaphor (see Taverniers, 2017). The example given in (1) provides an instance of 'smack dab' that is infrequent since it is instantiated here as a nominal but whether it is a single lexical item or not is a good question and so is whether or not its class or categorization is important (see discussion of transcategorization in Halliday and Matthiessen, 1999). Knowing its etymology helps in part, the OED lists it as an adverb ("smack dab" adv. 2016) but as speakers we know something about its frequency too. It is by far more frequently instantiated as in (2). If categorization or class of unit are important to the theory, as I think they are, then a more developed description of lexical representation is needed since the nature of the lexical item as instantiated form has serious impact on the theoretical model.

(1)in the course of 2 years I've gotten used to living in the equivalent of a medium-sized house right in the smack dab of Central London [source: enTenTen13 corpus using SketchEngine (Kilgarriff et al. 2014)]

(2) that puts your street address smack dab in the middle of the map [source: enTenTen13 corpus using SketchEngine (Kilgarriff et al. 2014)]

SFL is not alone in having to address these issues. In other areas of linguistics, such as in typological studies, "[t]he structure of the lexicon and its consequences for the language system have received much less attention" (Hengeveld, Rijkhoff and Siewierska, 2004:527). However, considering the structure of the lexicon forces the theory to engage with the associated assumptions. For example, in considering certain noun and verb pairs resulting from conversion, such as talk, walk, etc., Luuk (2010: 352) explains that "[i]n English ... tense-aspect-mood marking encodes predicate and determiners encode argument". The fluidity between nouns and verbs in these cases raises interesting questions about word classes as I have argued elsewhere Fontaine (2017a).

It could be argued that these stems are ambiguous in terms of their lexical class. This would force us to consider their lexical representation and we could ask whether they should be represented as the same lexeme or different (this could be considered in terms of polysemy or homonymy). Luuk (2010:362) argues that "they are neither nouns nor verbs but flexibles", i.e. polysemous lexemes. This suggests that in terms of lexical representation that there is one lexeme which encodes this type of flexibility but this is not true of all nouns or verbs so there must be something specific to this set (see Davies, 2004). According to Luuk, an implication of the assumption that accompanies alternative solutions for these items (either zero derivation or homophony) is that they "posit unnecessary hidden structure" (ibid., see also Don, 2005). At the moment, it would seem that SFL would assume homonymy but whether it would or would not consider such a solution is an unanswered question. In Fontaine (2017b), I argue for a preference for polysemy in lexical representation within the SFL framework. In considering the case of prepositions and particles, I show how this is not inconsistent with current SFL descriptions of English. While such representations may seem trivial at 
first sight, they have significant theoretical implications, which could send reverberations throughout the framework.

At this point, I would like to refer to a lexicological approach to lexical analysis as described by Hanks (2013), which has two main advantages in my view. The first is that it makes clear the distinction between the lexeme as meaning potential and the lexical item as instance. The second is that lexical meaning is seen as primarily collocational and in this sense seems very much in line with what Halliday was proposing in his 1961 paper.

In the remainder of this section, Hanks' (2013) approach to pattern analysis is adapted within SFL, drawing on the discussion of context given above and illustrated with a brief example to demonstrate how this might work.

In terms of lexical meaning, Hanks (2013:65) states his position as follows: "strictly speaking, words in isolation have meaning potential rather than meaning, and that actual meanings are best seen as events, only coming into existence when people use words, putting them together in clauses and sentences". This position needs to be reexpressed in more SFL terms. The reference to 'words in isolation' can be equated to Hanks' use of lemma, a term used in corpus linguistics to capture an abstract or uninflected form of a lexical item. This is equivalent to the term lexeme, which is typically used in psycholinguistic research. The terminology here is not very important but since lexeme has been used throughout this paper, we will continue to use it. What is significant is Hanks' view of the meaning potential of a lexeme. In this sense the semantics are represented as potential, as non-instantiated lexeme. The lexeme then has the full meaning potential, some of which (not necessarily all) is then activated when instantiated in text.

If we consider Hanks' example of BANK (2013:68-71), we can see that an account of word meaning needs to consider lexical semantics and this needs to be reflected in the lexical representation of the model. In considering various uses of BANK such as those in examples (3) and (4), both taken from Hanks (2013:68-69), the question arises whether the senses are related and if so whether "we go along with Pustejovsky, who would say that they are all part of the same 'lexical conceptual paradigm' (Pustejovsky, 1995)" (Hanks, 2013:69).

(3) $[\mathrm{He}]$ assaulted them in a bank doorway

(4) using a technique of photographic superimposition and then later a system of addition using banks of condensers

Hanks' solution is to identify the main semantic components as constituting the meaning potential of the lexeme. The semantic components for BANK include, according to Hanks (2013:70):

IS an institution.

IS a large building.

FOR storage.

FOR safekeeping of things.

FOR (esp.) safekeeping of finance/money.

CARRIES out transactions.

CONSISTS of a staff of people. 
Semantic components are "separate, combinable, exploitable entities" (ibid.). The instantiated lexical item such as in (9) or (10) will involve some combination of the components. Using semantic components this way enables a clearer sense of where the division between polysemy and homonymy lies. For example, BANK as in river bank does not share any semantic components with BANK in the examples which suggests separate lexemes, despite the similar form. For Hanks, a word gets its meaning from its use and in this sense, meaning is dynamic.

At this point it is useful to return to the concepts discussed above in relation to context. The question now is whether lexis can be said to be distributed within the SFL model in a similar way to context. If we accept that the meaning potential is construed by systems of language choice; the instance is construed by patterns of language use, then we might be willing to consider that the lexical item (instance) is construed by patterns of use and that the lexeme (meaning potential) is construed by systems of lexico-grammar. This does not imply that lexis is separate from lexicogrammar. The idea of meaning potential in terms of the lexeme is not far from Halliday and Matthiessen's view of items: "[t]he class of an item indicates in a general way its potential range of grammatical functions" (2014:76). If we are willing to entertain this idea as a proposal then it would suggest that rather than thinking of lexis only as most delicate grammar, we can think of lexis as most local context. This suggestion is illustrated in Fig. 3 below, which includes Halliday's (1991) diagram of language and context. The dotted line in the figure indicates that the proposal here concerns primarily the horizontal relation of instantiation but it is unclear at this point how this can be integrated into the vertical relation of realization, especially in terms of the lexicogrammar systems.

Such an approach might open up the theory of SFL to more lexicological concerns and potentially address both diachronic perspectives as well as the fluidity, flexibility and indeterminacy that we find in language. This proposal is only tentative and it would have to be examined and evaluated to see whether the modelling of lexis in

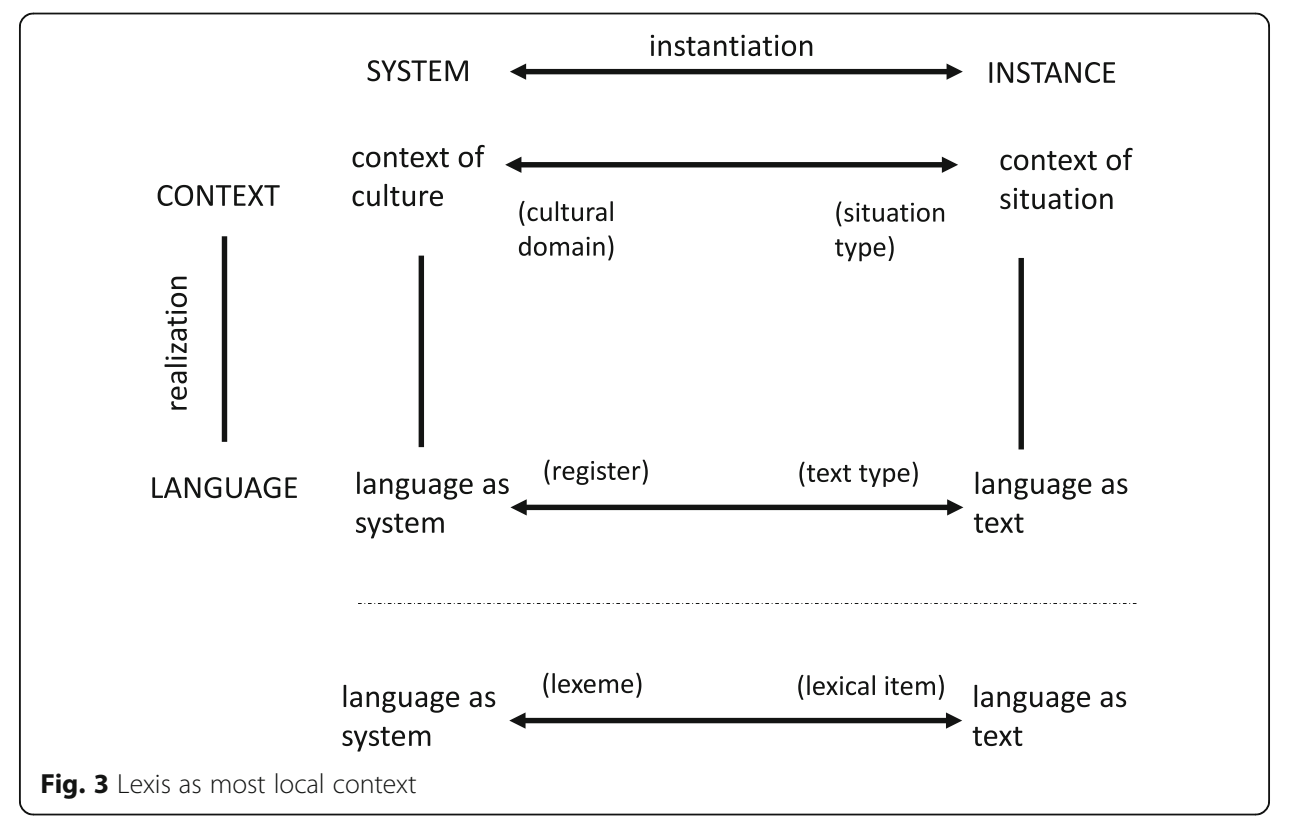


terms of context as suggested here may offer opportunities to account for shifting lexico-semantics. For example, according to García Velasco (2016: 940-941) "[usage] patterns may be modified or updated on the basis of new uses of the lexeme in different contexts. Meaning construction and sense modification is thus understood as the joint and cooperative activity of language users in verbal interaction". Context of culture and context of situation play an important role in this. The individual lexemes of the language constitute a semiotic repertoire that is available to the speaker and this must allow for innovative, meaning creating uses, or exploitations in Hanks' (2013) terminology.

\section{Concluding remarks}

In this paper, I set out to examine the relationship between context and lexicology. The way SFL talks about context is surprisingly similar to the way lexicologists talk about lexis. To date, very little attention has been given to developing an approach to lexical representation in SFL. This seems to be related to the top-down approach to lexicogrammar and to the view of lexis as most delicate grammar. The relationship between lexis and grammar cannot be denied, however some work from the bottom-up may contribute significantly to improving the overall account of the lexico-grammar. There is often a conflict for the analyst when trying to determine what the speaker has done with language and how the model used by the analyst can fit the instances of language being analysed. The speaker is generating language, while the analyst is parsing language, and doing so for very different reasons. In psycholinguistics, the problem of viewing these two processes (production vs understanding) as separate process is referred to by Pickering and Garrod (2013:347) as "the 'cognitive sandwich', a perspective that is incompatible both with the demands of communication and with extensive data indicating that production and comprehension are tightly interwoven". Reconciling the role of language producer and language understander is important for many reasons, not least of which is the development of a better tool for text analysis. Since it is not yet clear that the system networks are appropriate for the analyst or for the understander, as noted by Tucker (2009:424) above, this development is needed for those using the framework for analysis.

Even though SFL has an integrated lexico-grammar, it does not mean that there is no word meaning. As I have shown here, word meaning operates only in context and by introducing meaning potential at the lexical level, i.e. a mental lexicon, we open up the model to developments in lexicology both in terms of contributing and benefitting from research. This conceptualisation brings with it assumptions that must be tested but it does create an opportunity for building bridges with other theoretical approaches since it may be reasonable to talk about lexical meaning potential and how 'a meaning' is activated and how we can allow a more formal account of lexis into SFL theory. Lexis can be seen, rather than most delicate grammar, as most local context. Perhaps in some respects this is saying the same thing. As Halliday (1991:274) states, "the context for the meaning potential - for language as a system - is the context of culture. How do you construe this potential, and how do you use it when you've got it? You build it up, and you act it out, in the form of text". The meaning potential of a lexeme is construed, it is built up by its use and more specifically by its use in context. The meaning of a 
lexical item comes from its local context (lexeme), its collocations and the constructions or patterns (lexico-grammar) in which it appears. The view of lexis as most local context, where the lexeme (or lemma) is seen as a resource, or meaning potential, allows us to reconcile SFL with corpus-based approaches to lexico-grammar. As Hunston and Francis have suggested, it may be worth exploring what it would look like if "each bundle of system choices should end, not in a lexical item per se but in a 'unit of meaning"' (2000:28). The problem is, of course, determining where such a choice point should be within the system network and whether or not this would ultimately require a stratal distinction. The implementation of the theoretical ideas presented here is a work in progress and a concrete proposal is not yet ready for publication.

In closing, I follow Thompson (2015:26) when he says "everything we know about language suggests that structural configurations always take on a semiotic life of their own". This also applies to lexis. In SFL, one of the main approaches to working with variation is through context (especially context of situation or register). I have tried to reconcile these different perspectives by drawing on context to enable the representation of the semiotic life of words.

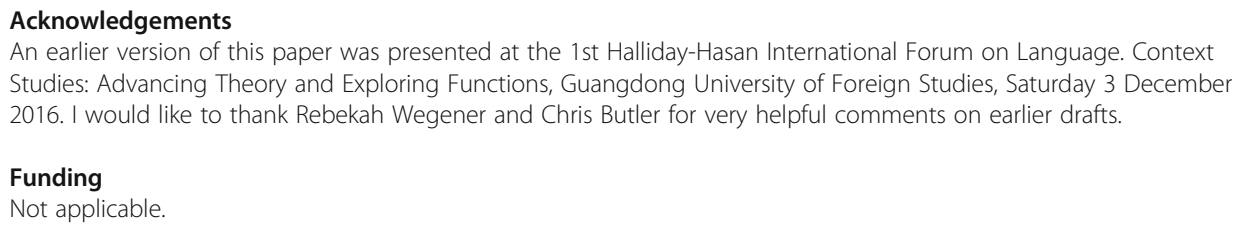

Availability of data and materials

Not applicable.

Authors' contributions

Not applicable.

Competing interest

The authors declare that they have no competing interests.

Ethics approval and consent to participate

The data collected for this paper comply with Cardiff University ethics procedures.

\section{Consent for publication}

Not applicable.

Received: 26 July 2017 Accepted: 14 November 2017

Published online: 22 November 2017

References

Bartlett, T. 2013. I'll manage the context: Context, environment and the potential for institutional change. In Systemic functional linguistics: Exploring choice, ed. L. Fontaine, T. Bartlett, and G. O'Grady, 342-364. Cambridge: Cambridge University Press.

Bartlett, T. 2017. Context in systemic functional linguistics: Towards scalar supervenience? In The Routledge handbook of systemic functional linguistics, ed. T. Bartlett and G. O'Grady, 375-390. London: Routledge.

Bednarek, M. 2008. Emotion talk across corpora. London: Palgrave Macmillan.

Bowcher, W. 2013. Material action as choice in field. In Systemic functional linguistics: Exploring choice, ed. L. Fontaine, T. Bartlett, and G. O'Grady, 318-341. Cambridge: Cambridge University Press.

Butler, C. 2009. Lexical phenomena in functional discourse grammar and systemic functional linguistics. In From will to well: Studies in Linguistics offered to Anne-Marie Simon-Vandenbergen, ed. S. Slembrouck, M. Taverniers, and M. van Herreweghe, 55-67. Gent: Academia press.

Butler, C. 2013. Systemic functional linguistics, cognitive linguistics and psycholinguistics: Opportunities for dialogue. Functions of Language 20 (2): 185-218.

Butt, D., R. Fahey, S. Spinks, and C. Yallop. 2000. Using functional grammar: An explorer's guide. 1st ed. Sydney: NCELTR, Macquarie University.

Cappelle, B., Y. Shtyrov, and F. Pulvermüller. 2010. Heating up or cooling up the brain? MEG evidence that phrasal verbs are lexical units. Brain and Language 115 (3): 189-201.

"smack-dab, adv." 2016 OED Online. Oxford University Press, December. Web. 15 February 2017.

Davidse, K. 2017. SFL and the clause: The experiential metafunction. In The Routledge handbook of systemic functional linguistics, ed. T. Bartlett and G. O'Grady, 79-95. London: Routledge. 
Davies, C. 2004. A Corpus-Based Investigation of Noun to Verb Conversion in English. Unpublished PhD Dissertation, University of Liverpool.

Don, J. 2005. On conversion, relisting and zero-derivation: A comment on Rochelle Lieber: English word-formation processes. SKASE Journal of Theoretical Linguistics 2 (2): 2-16.

Fawcett, R. 1994. On moving on on ontologies: Mass, count and long thing things. In Proceedings of the seventh international workshop on natural language generation, ed. D. McDonald, 71-80.

Fawcett, R. 2000. A theory of syntax for systemic functional linguistics. Amsterdam: John Benjamins.

Fawcett, R. 2014. The cultural classification of 'things': A system network for English noun senses. In: G. González, M. Ángeles, F. José Ruiz De mendoza Ibáñez \& F. Gonzálvez-García, eds., Theory and practice in functional-cognitive space. Amsterdam: John Benjamins, 53-84.

Fawcett, R., G. Tucker, and Y. Lin. 1993. How a systemic functional grammar works: The role of realization in realization. In New concepts in natural language generation, ed. H. Horacek and M. Zock, 114-186. London: Pinter.

Firth, J.R. 1957/1968. A synopsis of linguistic theory 1930-1955. In Selected papers of J.R. Firth 1952-1959, ed. F. Palmer, 168-205. London: Longman.

Fontaine, L. 2017a. Fluid boundaries and the categorization of nominal expressions. In Challenging boundaries in linguistics: Systemic functional perspectives. Aachen British and American studies series, ed. S. Neumann, R. Wegener, J. Fest, P. Niemietz, and N. Hützen, 19-38. Frankfurt am Main: Peter Lang Verlag.

Fontaine, L. 2017b. On prepositions and particles: A case for lexical representation in systemic functional linguistics. Word 63 (2): 115-135.

Fontaine, L., T. Bartlett, and G. O'Grady, eds. 2013. Systemic functional linguistics: Exploring choice. Cambridge: Cambridge University Press.

García Velasco, D. 2016. A flexible lexicon for functional discourse grammar. Linguistics 54 (5): 907-945.

Groom, N. 2005. Pattern and meaning across genres and disciplines: An exploratory study. Journal of English for Academic Purposes 4 (3): 257-277.

Halliday, M.A.K. 1961/2002. Categories of the theory of grammar. In On Grammar. Collected Works of MAK Halliday, vol. 1, ed. J. Webster, 95-105. London: Continuum.

Halliday, M.A.K. 1972/2013. With Herman Perret. 1972. In Interviews with M.A.K. Halliday: Language turned back on himself, ed. J. Martin, 1-40. London: Bloomsbury Academic.

Halliday, M.A.K. 1991/2007. The notion of "context" in language education. In Language and Education, Collected Works of MAK Halliday, vol 9, ed. J. Webster, 269-290. London: Continuum.

Halliday, M.A.K. 2013. Meaning as choice. In Systemic functional linguistics: Exploring choice, ed. L. Fontaine, T. Bartlett, and G. O'Grady, 15-36. Cambridge: Cambridge University Press.

Halliday, M.A.K., and R. Hasan. 1976. Cohesion in English. London: Cassell.

Halliday, M.A.K., and C. Matthiessen. 1999. Construing experience through meaning: A language-based approach to cognition. London: Cassell.

Halliday, M.A.K., and C. Matthiessen. 2014. Halliday's introduction to functional grammar. London: Routledge.

Hanks, P. 2013. Lexical analysis: Norms and exploitations. Cambridge, Mass: MIT Press.

Hasan, R. 1996. The grammarian's dream: Lexis as most delicate grammar. In Ways of saying, ways of meaning: Selected papers of Ruqaiya Hasan, ed. C. Cloran, D. Butt, and G. Williams, 73-103. London: Cassell.

Hasan, R. 2009. The place of context in a systemic functional model. In Continuum companion to systemic functional linguistics, ed. M.A.K. Halliday and J. Webster, 166-189. London and New York: Continuum.

Hasan, R. 2013. Choice, system, realization: Describing language as meaning potential. In Systemic functional linguistics: Exploring choice, ed. L. Fontaine, T. Bartlett, and G. O'Grady, 269-299. Cambridge: Cambridge University Press.

Hengeveld, K., J. Rijkhoff, and A. Siewierska. 2004. Parts-of-speech systems and word order. Journal of Linguistics 40: 527-570.

Hunston, S., and G. Francis. 2000. Pattern grammar: A corpus-driven approach to the lexical grammar of English. Amsterdam: John Benjamins.

Kilgarriff, A. et al. 2014. The Sketch Engine: ten years on. Lexicography, 1, pp. 1-30. [online] Sketchengine.co.uk. Available at: http://www.sketchengine.co.uk [Accessed 19 Feb. 2017].

Luuk, E. 2010. Nouns, verbs and flexibles: Implications for typologies of word classes. Language Sciences 32 (3): 349-365.

Martin, J. 1992. English text: System and structure. Amsterdam: John Benjamins.

Martin, J. 2016. Meaning matters: A short history of systemic functional linguistics. Word 62 (1): 35-58.

Matthiessen, Christian M.I.M. 2014. Extending the description of process type within the system of transitivity in delicacy based on Levinian verb classes. Functions of Language, Vol. 21:2, pp. 139-175.

Matthiessen, C. 2015. Modelling context and register: The long-term project of registerial cartography. Letras, Santa Maria 25 (50): 15-90.

Matthiessen, C., and J. Bateman. 1991. Text generation and systemic-functional linguistics: Experiences from English and Japanese. London: Frances Pinter Publishers and St. Martin's Press

Mel'čuk, I. 1981. Meaning-text models: A recent trend in soviet linguistics. Annual Review of Anthropology. 10: 27-62.

Moon, R. 1997. Vocabulary connections: Multi-word items in English. In Vocabulary: Description acquisition and pedagogy, ed. N. Schmitt and M. McCarthy, 40-63. Cambridge: Cambridge University Press.

O'Donnell, M., H. Cheng, and J. Hitzeman. 1998. Integrating referring and informing in NP planning. Proceedings of the ColingACL'98 workshop on the computational treatment of Nominals, august 16, 1998, 46-55. Montréal: Université de Montreal.

Pickering, M., and S. Garrod. 2004. Toward a mechanistic psychology of dialogue. Behavioral and Brain Sciences 27 (2): 169-225.

Pickering, M., and S. Garrod. 2013. An integrated theory of language production and comprehension. Behavioral and Brain Sciences 36 (4): 329-347.

Plum, G. (1998) Text and Contextual Conditioning in Spoken English: A genre-based approach. Nottingham: University of Nottingham (Monographs in Systemic Linguistics 10).

Pustejovsky, J. 1995. The generative lexicon. Cambridge. MA: MIT Press.

Rijkhoff, J., and E. van Lier. 2013. Flexible word classes: A typological study of underspecified parts-of-speech. Oxford: Oxford University Press.

Sanders, T., and H. Pander Maat. 2006. Cohesion and coherence: Linguistic approaches. In Encyclopedia of language and linguistics, ed. K. Brown, vol. 2, 2nd ed., 591-595. Amsterdam: Elsevier.

Steels, L., ed. 2011. Design patterns in fluid construction grammar. Amsterdam: John Benjamins. 
Steels, L. \& J. De Beule. 2006. A (very) brief introduction to fluid construction grammar Proceedings of the 3rd Workshop on Scalable Natural Language Understanding, New York City, June 2006, pp. 73-80.

Taverniers, M. 2017. Grammatical metaphor. In The Routledge handbook of systemic functional linguistics, ed. T. Bartlett and G. O'Grady, 354-372. London: Routledge.

Thompson, G. 2015. Pattern grammar and transitivity analysis. In Corpora, grammar and discourse, ed. M. Charles, N. Groom, and S. John, 21-42. Amsterdam: John Benjamins.

Tucker, G. 1996. Cultural classification and system networks: A systemic functional approach to lexis. In Meaning and form: Systemic functional interpretations. Meaning and choice in language: Studies for Michael Halliday, ed. M. Berry, C. Butler, R. Fawcett, and G. Huang, 533-536. Norwood, NJ: Ablex.

Tucker, G. 1998. The lexicogrammar of adjectives: A systemic functional approach to lexis. London: Cassell Academic.

Tucker, G. 2006. Between grammar and lexis: Towards a systemic functional account of phraseology. In Continuing discourse on language: A functional perspective, ed. C. Matthiessen, R. Hasan, and J. Webster, 954-977. London: Equinox.

Tucker, G. 2009. In search of the grammarian's dream: Locating lexis in a systemic functional grammar. In From will to well: Studies in linguistics offered to Anne-Marie Simon-Vandenbergen, ed. S. Slembrouck, M. Taverniers, and M. van Herreweghe, 415-426. Gent: Academia Press.

Wegener, R. 2011. Parameters of context: From theory to model and application. Unpublished PhD thesis. Sydney: Macquarie University.

Wray, A. 2014. Why are we so sure we know what a word is? In The Oxford handbook of the word, ed. J. Taylor, 725750. Oxford: Oxford University Press.

Submit your manuscript to a SpringerOpen ${ }^{\circ}$ journal and benefit from:

- Convenient online submission

- Rigorous peer review

- Open access: articles freely available online

- High visibility within the field

- Retaining the copyright to your article

Submit your next manuscript at $>$ springeropen.com 